\title{
Glycine Transporter Type I (GlyT1) Inhibitor, Bitopertin: A Journey from Lab to Patient
}

\author{
Emmanuel Pinard\$^, Edilio Borroni, Annette Koerner, Daniel Umbricht, and Daniela Alberati
}

§SISF-SCS Senior Industrial Investigator Award 2017

\begin{abstract}
Glycine transporter-1 (GlyT1) inhibition has been extensively studied both in pharmaceutical companies and academic institutions primarily as a potential new approach to treat schizophrenia, a severe and chronic mental illness. More recently, preclinical results have suggested that this approach could also have therapeutic potential for CNS disorders beyond schizophrenia as well as for non-CNS indications. Over the past 17 years, Roche has been a key player in the GlyT1 field with the discovery and development of bitopertin, the most advanced GlyT1 inhibitor to date and the only one which completed Phase III clinical studies for schizophrenia. In this article, we relate the eventful journey of the discovery and development of bitopertin, from project initiation in 2001 to its evaluation today in patients suffering from beta-thalassemia, a monogenic hereditary haematological disorder.
\end{abstract}

Keywords: Beta-thalassemia $\cdot$ GlyT1 $\cdot$ Medicinal chemistry $\cdot$ PET $\cdot$ Schizophrenia $\cdot$ Transporter

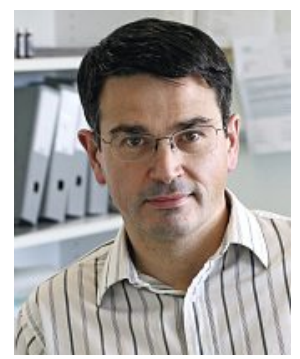

Emmanuel Pinard joined F. HoffmannLa Roche in Basel in 1995 after completing his PhD in France (University P. et M. Curie in Paris, Prof. A. Marquet) and a postdoctoral fellowship in the USA (Ohio State University, Prof. L. A. Paquette). He has been working in the medicinal chemistry department in the area of CNS and since 2001 as discovery chemistry project leader. He holds more than 100 patents and publications.

\section{Introduction}

Schizophrenia is a severe and chronic mental disorder with a world-wide life time prevalence ranging from $0.5 \%$ to $1 \%$. The symptoms of schizophrenia are broadly categorised as positive symptoms (hallucinations, delusions), negative symptoms (social withdrawal, lack of motivation and emotional reactivity) and cognitive impairment (working memory, attention and executive function deficits). In 2001, when we started our GlyT1 program, the leading approved treatments for schizophrenia were, and in fact, still are, today, the antipsychotics that belong to the family of dopamine D2 receptor antagonists. These therapies are efficacious in the management of positive symptoms, however, they have no or little effects on negative symptoms and cognitive impairment. The limited impact on negative symptoms is in particular an issue since these symptoms are recognised as one of the main causes of social and occupational dysfunction for patients with schizophrenia. Thus, the suboptimal efficacy profile observed with the available dopaminergic-based drugs certainly called for the identification of novel approaches for schizophrenia.[1] During the mid-late 1990s, solid evidence started to accumulate suggesting that the hypofunction of N-methyl-D-aspartate receptors (NMDA-R) may play an important role in the pathophysiology of schizophrenia. ${ }^{[2]}$ The most prominent evidence supporting this hypothesis was the observation that selective NMDA-R blockers like phenylcylohexylpiperidine (PCP), and ketamine were able to reproduce in healthy subjects the positive, negative and cognitive symptoms of schizophrenia as well as to worsen existing symptoms in schizophrenic patients. ${ }^{[2]}$ In addition, this hypothesis was supported by a number of both animal and genetic studies. ${ }^{[2]}$ These elements clearly suggested that pharmacological enhancement of NMDA-R activity could represent a promising novel strategy for the management of schizophrenia that could potentially confer greater therapeutic effects than the available antipsychotic drugs. ${ }^{[3]}$ In the late 1990s, the neurotransmitter glycine was identified as an obligatory co-agonist and allosteric positive modulator at the NMDA-R acting at the glycine binding site on the GluN1 subunit (Fig. 1). ${ }^{[4]}$ A logical approach to potentiate the activity of the NMDA-R which we initially followed, was to look for potent and selective NMDA glycine site agonists having superior brain penetration and PK profiles than glycine itself. However, this pharmacological approach did not prove successful, very likely due to the high polarity and to the restricted size of the NMDA glycine binding site. An alternative pharmacological approach we decided to start exploring in 2001, was the elevation of extracellular concentrations of the co-agonist glycine in the brain through inhibition of glycine uptake mediated by GlyT1, a selective transporter of glycine known to be co-expressed with NMDA-R..5] GlyT1 belongs to the $\mathrm{Na}^{+} / \mathrm{Cl}^{-}$-dependent family of neurotransmitter transporters, which in-

\footnotetext{
${ }^{*}$ Correspondence: Dr. E. Pinard
Roche Pharmaceutical Research and Early Development (pRED)

Roche Innovation Center Basel

Medicinal Chemistry Department

124 Grenzacherstr

E-mail: emmanuel.pinard@roche.com
} 
cludes GlyT2, another selective transporter of glycine which shares $50 \%$ amino-acid similarity with GlyT1, as well as the wellknown GABA and monoamine transporters such as the serotonin and dopamine transporters: SERT and DAT. GlyT1 has a widespread distribution in the brain and is expressed both on presynaptic neurons and on astrocytes of NMDA synapses (Fig. 1). ${ }^{[5]}$ Support for the GlyT1 inhibitor approach and therefore for our project, came later, in 2004, when Tsai et al. reported positive results of clinical studies showing that a high dose ( $2 \mathrm{~g} /$ day) of sarcosine (N-methyl glycine), a weak GlyT1 inhibitor, had beneficial effects on positive, negative and cognitive symptoms in schizophrenic patients when administered on top of conventional schizophrenia drugs. ${ }^{[6]}$

\section{Discovery of Bitopertin}

In 2001, the year of the start of our program, the field of GlyT1 started to evolve in the scientific community. This was mainly due to the publication of the very first potent and selective GlyT1 inhibitors: Org-24598[7] and ALX-5407 (Fig. 2). ${ }^{[8]}$ Interestingly, from a structural point of view, these first inhibitors were both sarcosine-based derivatives, carrying large hydrophobic fragments reminiscent of those found in known inhibitors of other members of the same transporter family, namely SERT.

These exciting reports appeared to have trigged the initiation of GlyT1 inhibitor programs at many other pharmaceutical companies, some of them focusing their medicinal chemistry activities on the sarcosine chemotype, as indicated by articles or patents published a few years later. ${ }^{[5 b, c]}$ At Roche, we decided not to embark on a competitor-driven chemistry program on sarcosines. This decision was mainly based on the observation that single administration of low, in vivo pharmacologically active doses of Org-24598 or ALX-5407 in rodents caused a range of severe side effects including ataxia, motor and respiratory dysfunction. At the time of this decision, we did not know whether these effects were target or class related. Later studies published by others suggested that the side effects observed with these early sarcosines were in fact likely due to their very slow dissociation binding kinetics (i.e. slow $\mathrm{k}_{\text {off }}$ ) making them pseudo-irreversible inhibitors of GlyT1. [9] These sarcosines have nevertheless been very useful pharmacological tools to us for the establishment and validation of both in vitro and in vivo assays of the screening cascade we used during our lead identification and optimisation program (Fig. 3).

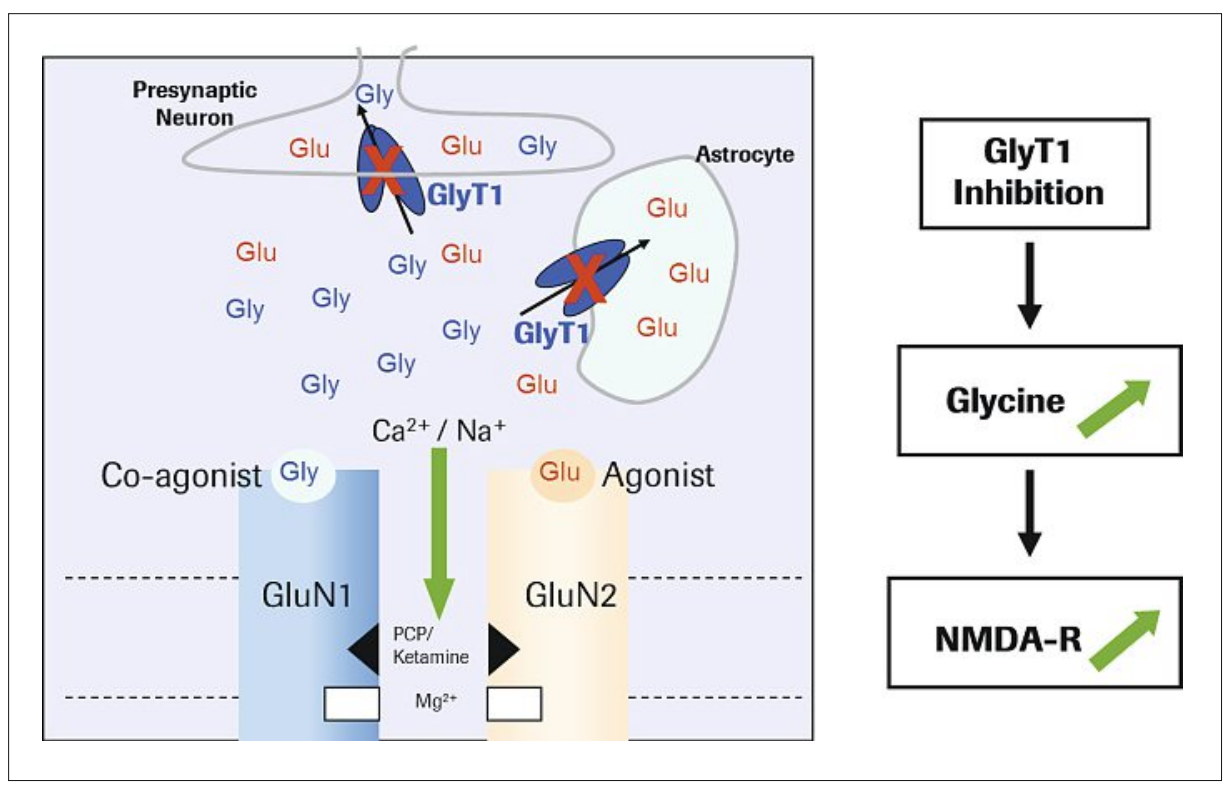

Fig. 1. GlyT1 inhibition: a mechanism to potentiate NMDA-R activity through local increase of the level of the co-agonist glycine.

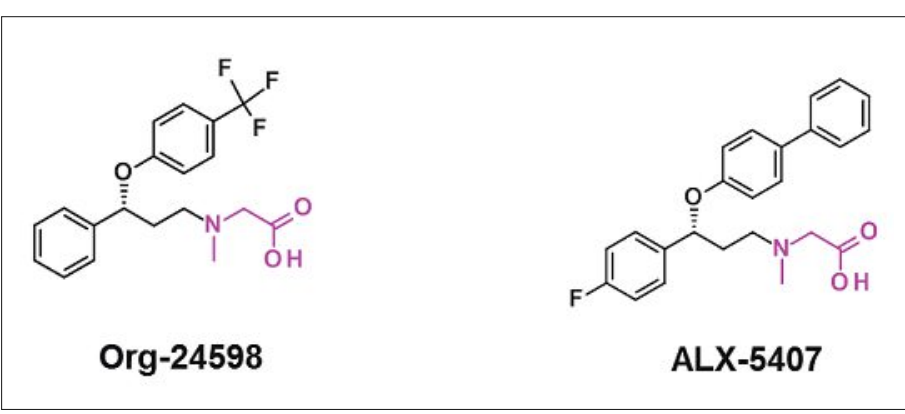

Fig. 2. Sarcosine derivatives: the first potent and selective described GlyT1 inhibitors.

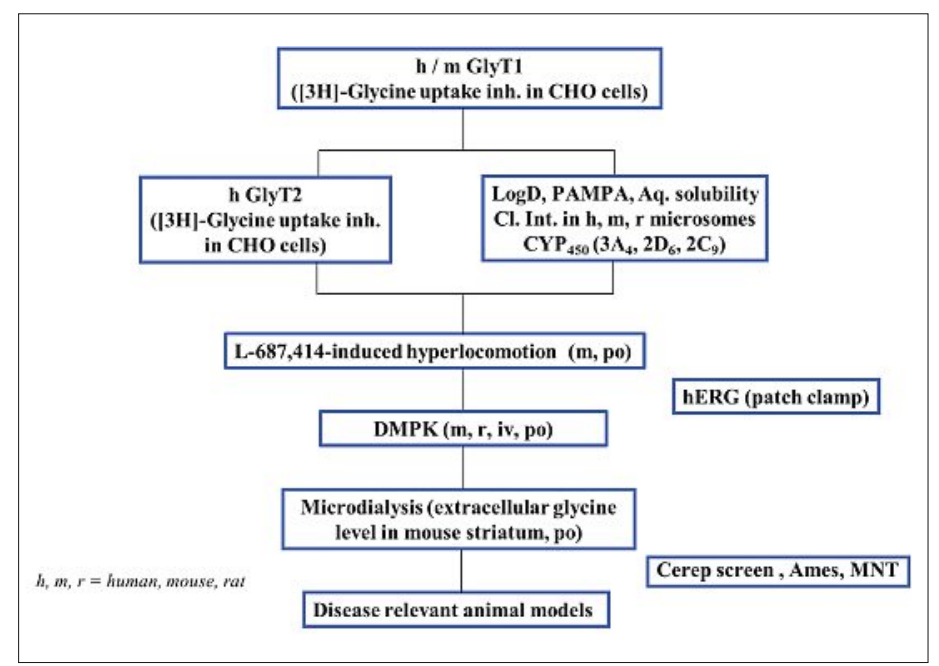

Fig. 3. Screening cascade used during the lead identification/ optimization phase.

Noteworthy, as a first-line model for the in vivo screening of our compounds, we have utilised the L-687,414 induced hyperlocomotion assay, a novel procedure established in the project which we found able to reliably and rapidly detect the in vivo activity of GlyT1 inhibitors. ${ }^{[10]}$ In this assay, the activity of compounds are determined by measuring their ability to prevent the hyperlocomotion induced in mice by L-687,414, a selective brain penetrant an- tagonist at the glycine site of the NMDA receptor. ${ }^{[11]}$ This effect observed with GlyT1 inhibitors likely results from the increased extracellular level of glycine in the brain and the subsequent displacement of L-687,414 from the glycine binding site of the NMDA receptor. This pharmaco-dynamic in vivo method proved to have excellent throughput and turned out to be essential for extremely efficiently guiding our discovery program. Active compounds 
in this model were then tested in the more labour intensive and time consuming microdialysis procedure to evaluate their effect on extracellular glycine levels in mouse brain.

\subsection{HTS Campaigns: Identification of Benzoylpiperazine Hit Series}

With the aim to identify non-sarcosine-based GlyT1 inhibitor scaffolds, an HTS campaign of the Roche compound collection (of about 700,000 compounds) was performed early 2001 using a $[3 \mathrm{H}]$-glycine uptake inhibition assay performed with CHO cells transfected with hGlyT1. This campaign successfully delivered a number of structurally diverse hit classes showing, for some, excellent GlyT1 potency (Fig. 4). However, our optimisation activities on these scaffolds did not lead to compounds with sufficiently attractive overall profiles and these classes had to be abandoned. ${ }^{[12]}$ The most common issues we encountered in these series were: low selectivity against the GlyT2 isoform, high microsomal clearance as well as off-target activities.

The lead identification challenges we were faced with the hit series identified from the 2001 HTS, led us in 2002 to perform a follow-up screen on new compounds (about 60,000 ) that had been added to the Roche collection since the first campaign. Among the validated hits identified, the benzoylpiperazine compound $\mathbf{1}^{[13]}$ (Fig. 5) carrying a morpholine and a nitro group at positions 2 and 5 respectively on the benzoyl part and a 2-fluoro-4-acetophenone group on the piperazine nitrogen immediately attracted our attention not only by its excellent GlyT1 potency ( $15 \mathrm{nM})$ but also by its chemical simplicity and tractability. Strikingly, this hit was a singleton that originated from a purchased benzoylpiperazine library containing more than 900 members A literature and patent search revealed that although structurally simple, 2,5-disubstituted benzoylpiperazine chemotypes, such as $\mathbf{1}$ had practically not been described in medicinal chemistry programs earlier. Thus, the identification of $\mathbf{1}$ opened to us an exciting patent free chemical space in which we could operate. In addition, further profiling revealed, that, $\mathbf{1}$ had positive characteristics: a good selectivity against GlyT2, no off-target activity in a Cerep panel screen (86 targets), good molecular properties with a medium $\log \mathrm{D}$ and high permeability (PAMPA) as well as in vivo activity in our L-687,414-induced hyperlocomotion screening assay after ip administration $\left(\mathrm{ID}_{50}: 20 \mathrm{mg} / \mathrm{kg}\right.$ ) indicating CNS penetration. On the more negative side, 1 displayed a pronounced microsomal clearance resulting in low oral bioavailability and was thus devoid of oral activity in our in vivo screening model. Another limitation was the presence of the nitro group, an ele-

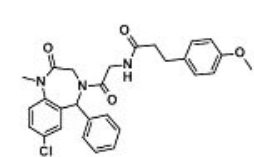

Benzodiazepinones $0.15 \mu \mathbf{M}$

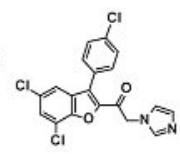

Benzofurans $0.068 \mu \mathrm{M}$

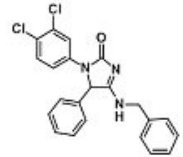

Imidazolidinones $0.076 \mu \mathbf{M}$

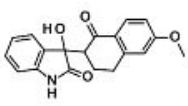

Indolinones

$0.103 \mu \mathrm{M}$

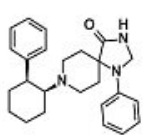

Spiropiperidines

$0.026 \mu \mathrm{M}$

hGLVTT $E C_{50}$

Fig. 4. Hits from the first HTS campaign.

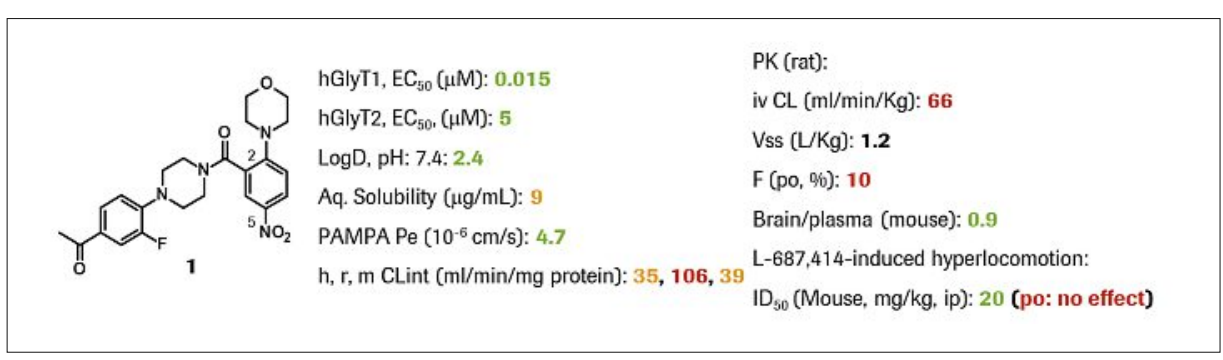

Fig. 5. Structure and profile of benzoylpiperazine HTS hit 1.

ment often causing safety concern due to its well-documented mutagenic and carcinogenic potential. The overall attractive profile of hit 1 together with its high chemical tractability led us to decide early 2003 to focus our medicinal chemistry program exclusively on this benzoylpiperazine class.

\subsection{From HTS Hit 1 to Bitopertin}

The key drug design steps that led to the identification of bitopertin are indicated in Fig. $6 .{ }^{[13,14]}$ The first goal was to identify drug-like and safe replacements for the nitro group and to establish the SAR at the morpholine and at the left-hand aromatic regions. A detailed evaluation of a diverse set of substituents at position 5 revealed that the methylsulfone group (compound 2) behaved as an excellent substitute for the nitro group. Indeed, with this polar electron withdrawing group in place, not only was the GlyT1 potency retained, but also the activity at GlyT2 was abolished, the solubility was improved and the intrinsic clearance in particular in rat microsomes was reduced (Fig. 7). Therefore, methylsulfone soon constituted our preferred group at position 5 on our benzoylpiperazine scaffold. At the morpholine position, a broad diversity of substituents was found to be tolerated such as alkoxy, alkyl amino, aromatic, heteroaromatic, cycloalkyl and heterocycloakyl groups provided that these groups had an adequate size and lipophilicity. At the lefthand aromatic moiety, a polar or lipophilic electron withdrawing group in para position was required for GlyT1 activity such as $-\mathrm{CF}_{3},-\mathrm{CN},-\mathrm{SO}_{2} \mathrm{Me}$. With this critical SAR information in hand, the first round of optimization was then performed by combining best elements identified at the mor- pholine and left-hand aryl moieties keeping the methylsulfone group at position 5. This work, which was done mostly using parallel synthesis, allowed the rapid production of a large number $(>100)$ of highly potent analogues. The profiling of this diverse set of compounds in our battery of multi-dimensional optimisation (MDO) assays, ${ }^{[15]}$ to measure their molecular properties and microsomal clearances as well as the assessment of their effects in our rapid L-687,414 hyperlocomotion screening model, quickly indicated that analogues having an alkoxy group at the initial 'morpholine' region had the best overall profile with balanced molecular properties, high metabolic stability and oral bioavailability. A representative example from this promising subclass was the cyclopropylmethylenoxy-substituted compound 3 (GlyT1 $\mathrm{EC}_{50}=16 \mathrm{nM}$ ) having a 2-F, 4-CN-phenyl moiety on the left-hand side. Compound $\mathbf{3}$ displayed an excellent mouse oral bioavailability $(\mathrm{F}=100 \%)$ and a good oral effect in the L-687,414-based assay $\left(\mathrm{ID}_{50}=3 \mathrm{mg} / \mathrm{kg}\right.$ ) despite demonstrating a fairly low brain penetration (brain/ plasma: 0.1). Moreover, in the microdialysis procedure performed in mice, 3 produced a robust 2.3-fold glycine increase over basal level at an oral dose of $10 \mathrm{mg} /$ $\mathrm{kg}$ thus offering the mechanistic proof that compounds from our series such as $\mathbf{3}$ were acting in vivo as GlyT1 inhibitors. In addition, $\mathbf{3}$, like all compounds from the alkoxy subclass, showed no significant inhibition of the major drug metabolizing $\mathrm{CYP}_{450}$ enzymes and was devoid of off-target activities in a Cerep selectivity screen. However, 3 could not be developed further because of a pronounced hERG inhibitory activity $\left(\mathrm{IC}_{50}: 0.6 \mu \mathrm{M}\right)$. 
The evaluation of a diverse set of alkoxy moieties revealed that a significant decrease of hERG activity was observed upon replacing the cyclopropylmethylenoxy group of $\mathbf{3}$ with shorter substituents such as the isopropyloxy or trifluoisopropyloxy group (compound 4). Moreover, with the trifluoisopropyloxy group in place, a simultaneous improvement in brain penetration (brain/plasma: 0.25 ) and in in vivo efficacy $\left(\mathrm{ID}_{50}=1 \mathrm{mg} / \mathrm{kg}\right.$ po) was observed. Thus, the trifluoisopropyloxy group having positive effects on CNS penetration plus in vivo and hERG profiles emerged as the best substituent among the set of alkoxy residues we explored. Next, keeping this group in place, a further improvement in brain penetration was seen upon replacing the polar $p$-nitrile substituent by the more lipophilic $p$-trifluoromethyl group at the left-hand aromatic moiety (compound $\mathbf{5}$, brain/plasma 1.15). This good result was explained by the reduced polar surface area (PSA) and the increased lipophilicity ( $\log \mathrm{P}$ ) of $\mathbf{5}$ compared to the nitrile substituted compound 4 . In fact, the
CNS penetration in our series was generally very well described by using solely PSA and $\operatorname{cog} \mathrm{P}$ as descriptors. ${ }^{[14]}$ In addition to its improved brain penetration, trifluoromethylated compound $\mathbf{5}$ exhibited a significantly reduced hERG activity $\left(\mathrm{IC}_{50}=6.9 \mu \mathrm{M}\right)$ compared to 4 . Next, a further improvement in hERG selectivity profile could be efficiently reached upon exchanging the left-hand aromatic nucleus of 5 with more polar heteroaromatic systems as seen with trifluoromethyl-pyridyl substituted compound $\mathbf{6}$ displaying a hERG $\mathrm{IC}_{50}$ of $20 \mu \mathrm{M}$. However, likely because of its decreased clogP and increased PSA, 6 displayed a reduced CNS penetration (brain/plasma: 0.2) and hence a lower in vivo activity $\left(\mathrm{ID}_{50}=5 \mathrm{mg} / \mathrm{kg} \mathrm{po}\right)$. This deficiency could be nicely addressed upon inserting at the position 3 of the pyridyl unit of 6 a fluorine atom. The resulting potent compound 7 exhibited indeed an improved brain penetration (brain/plasma: $0.5)$ and consequently a superior in vivo effect $\left(\mathrm{ID}_{50}=1 \mathrm{mg} / \mathrm{kg}\right.$ po) while keeping the $\mathrm{hERG}$ activity at low level $\left(\mathrm{IC}_{50}=\right.$
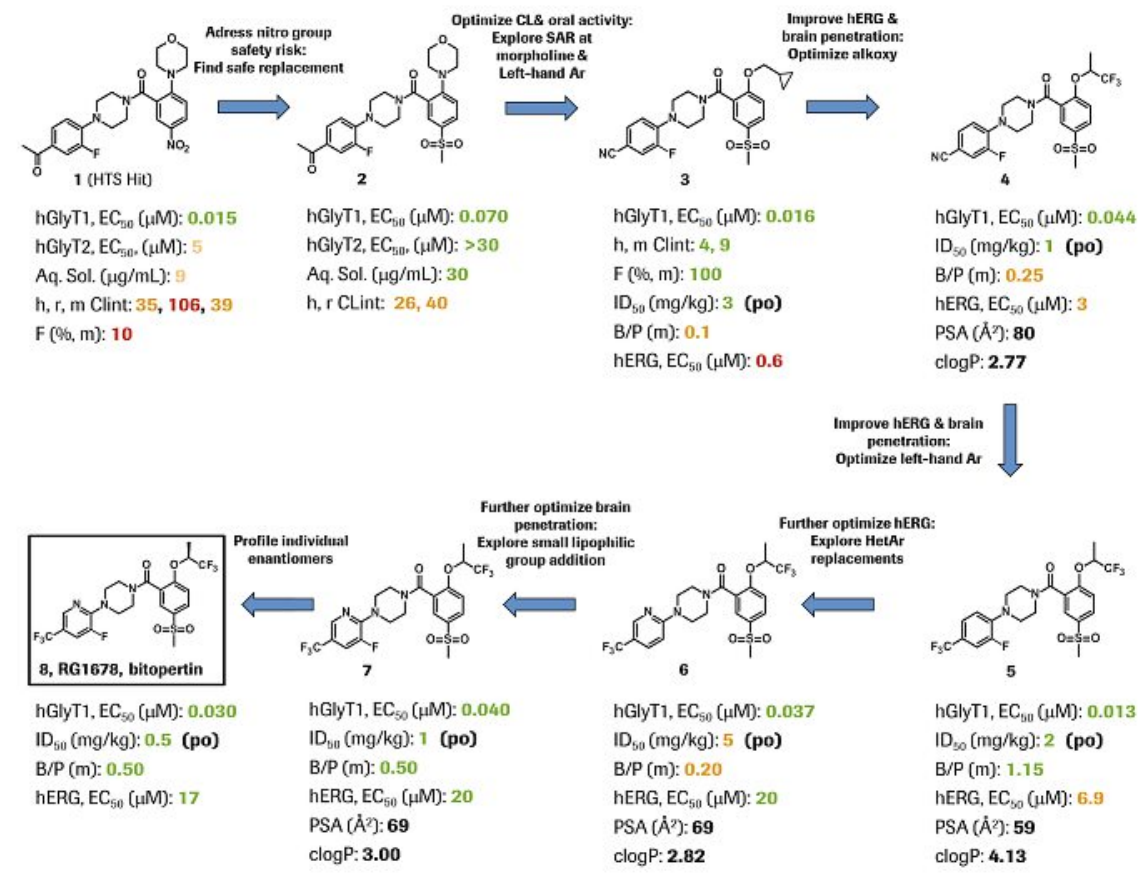

Fig. 6. From benzoylpiperazine HTS hit 1 to bitopertin: Key drug design steps.

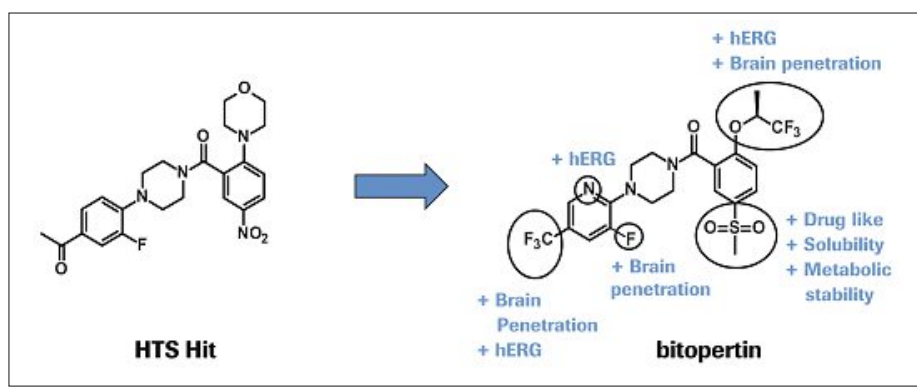

$20 \mu \mathrm{M})$. The alternative substituents that were evaluated at the same position (i.e. methyl, chlorine) led to compounds having much inferior profiles, showing here the unique beneficial effect that a fluorine atom can have on compound properties. Best profile was achieved with the $(S)$ configurated enantiomer 8 showing, in comparison with the $(R)$-enantiomer, a higher GlyT1 potency $\left(\mathrm{IC}_{50}=30 \mathrm{nM}\right)$ and a superior in vivo efficacy that reached an $\mathrm{ID}_{50}$ as low as $0.5 \mathrm{mg} / \mathrm{kg}$ po while retaining a low hERG activity $\left(\mathrm{IC}_{50}=17 \mu \mathrm{M}\right)$. $^{[14]}$ Further profiling revealed that $\mathbf{8}$ had an excellent selectivity profile against the GlyT2 isoform $\left(\mathrm{IC}_{50}>30 \mu \mathrm{M}\right)$ and was devoid of off-target activities in a Cerep selectivity screen. In vivo pharmacokinetic studies in our two preclinical species: rat and monkey[14] showed that $\mathbf{8}$ had a low plasma clearance, a good oral bioavailability as well as a favourable half-life. Using a Physiologically Based Pharmacokinetic modelling (PBPK) approach, ${ }^{[16]}$ a terminal half-life of about $40 \mathrm{~h}$ in the human species was predicted for $\mathbf{8}$ supporting for this compound a once-daily dosing regimen. As a result of its highly promising early preclinical profile, compound $\mathbf{8}$, named first RG1678 and later on bitopertin, was selected early 2004 as a clinical candidate. In summary, starting from our benzoylpiperazine hit structure (Fig. 7), the discovery process we followed led us, in just one year, to a highly optimised molecule in which each functional group around the central core has a specific role and nicely contributes to the overall excellent compound profile. The availability of a fast in vivo screening assay together with the capacity to profile our compounds in parallel in in vitro MDO assays have been essential to design and to identify RG1678, fulfilling our desired compound profile within a short timeframe.

\section{Discovery of Roche GlyT1 PET Tracer $\left[{ }^{11} \mathrm{C}\right] \mathrm{RO} 05013853$}

To support the clinical development of bitopertin, we started mid-2004 a program aiming at discovering a suitable PET tracer for GlyT1. A PET ligand is an important and powerful biomarker agent that allows the determination of the relationship between the drug dose, plasma exposure, target occupancy and therapeutic effect. At that time, no such ligand had been reported for GlyT1. Our search began with the profiling of tritiated-bitopertin in in vitro autoradiography in rat brain slices. Unfortunately, it did not behave well in this experiment. A strong non-specific binding due likely to a relatively high lipophilicity (clogP: 3 ) and high protein binding ( $97 \%$ in the rat) was 
observed. These results led us to profile, as potential PET candidates, compounds from a structurally related series, named benzoylisoindolines, a class we had identified during the course of our medicinal program following a cyclic amine screening approach (Fig. 8).[17]

In this series, a larger structural diversity for GlyT1 activity was observed on the left-hand region compared to the parent benzoylpiperazine series, which led to the identification of a number of highly potent, CNS penetrant molecules having reduced lipophilicity and protein binding. ${ }^{[17]}$ Among those was RO4993850/RG7118, (Fig. 8) a molecule that in fact reached and completed phase I clinical studies in healthy volunteers. ${ }^{[18]}$ RO4993850 as well as two tetrahydropyranyl-substituted-isoindolines, RO5013852 and RO5013853 (Table 1), were tritiated and profiled in in vivo autoradiography experiments. The selection of the latter two compounds was also based on the fact that both demonstrated a high iv clearance, a property known to be favourable for a PET radiotracer (Table 1). Gratifyingly, both tetrahydropyranyl analogues exhibited, as wished, robust specific signals, superior to those measured for bitopertin and RO4993850, two low clearance compounds. RO5013852 and RO5013853 were thus selected as potential PET candidates.

For the synthesis of our two PET candidates, we envisioned to label the methyl-sulfone substituent with the shortlived ${ }^{11} \mathrm{C}$ radioisotope (half-life of about 20 min.). In collaboration with Prof. Dean F. Wong and Robert F. Dannals from the Johns Hopkins University School of Medicine, this goal could be achieved through the incorporation of the radioisotope in the final step through methylation of an easily accessible sodium sulfinate precursor with $\left[{ }^{11} \mathrm{C}\right]$-methyliodide (Fig. 9). ${ }^{19]}$

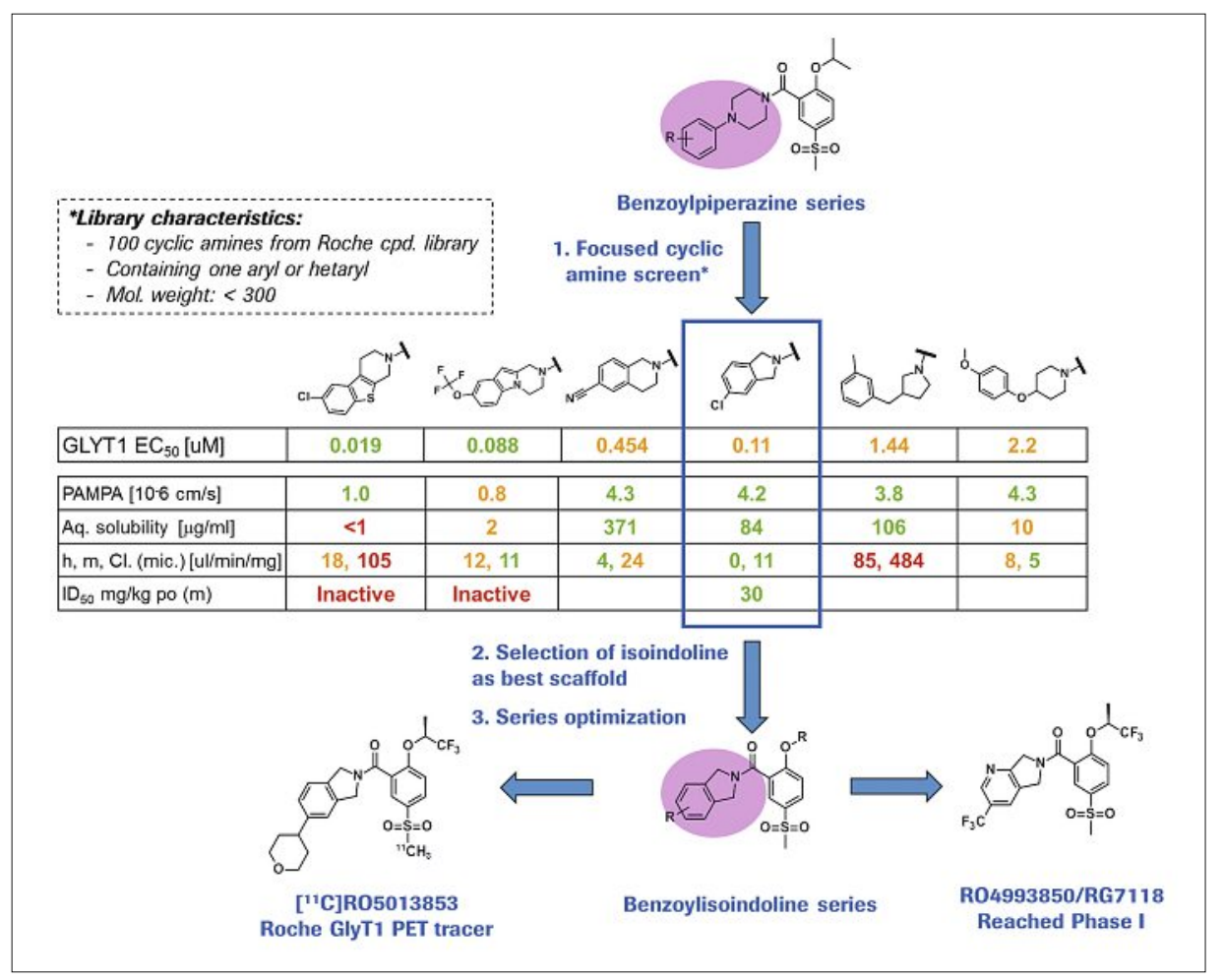

Fig. 8. From benzoylpiperazine to benzoylisoindolines: Expanding the GlyT1 chemistry and clinical toolbox.

In a subsequent PET study done in baboon, $\left[{ }^{11} \mathrm{C}\right] \mathrm{RO} 05013853$ showed a stronger specific signal and better brain uptake than $\left[{ }^{11} \mathrm{C}\right] \mathrm{RO5013852}$ and was thus selected for further characterisation. Furthermore, blockade experiment with bitopertin showed that $\left[{ }^{11} \mathrm{C}\right] \mathrm{RO} 5013853$ was suitable for receptor occupancy studies. In the baboon, $50 \%$ receptor occupancy in the thalamus was achieved for bitopertin at the plasma concentration $\left(\mathrm{Occ}_{50}\right)$ of $332 \mathrm{ng} / \mathrm{ml}$. $^{20]}$ In PET studies performed in human (healthy volunteers), $\left[{ }^{11} \mathrm{C}\right] \mathrm{RO} 05013853$ performed equally well.
[21] At steady state, bitopertin produced, in the pons, thalamus, and cerebellum, a dose-dependent engagement of the GlyT1 target with plasma Occ $_{50}$ of about $190 \mathrm{ng} / \mathrm{ml}$, at an estimated dose $\left(\mathrm{ED}_{50}\right)$ of $15 \mathrm{mg} .{ }^{[22]}$ In summary, with $\left[{ }^{11} \mathrm{C}\right]$ RO5013853, we successfully discovered and developed a valid PET tracer for the GlyT1 target. This tracer has been an important tool for supporting clinical dose selection as well as for interpreting the dose/efficacy relationship observed with bitopertin both in preclinical and clinical studies (vide infra).

Table 1. Properties of PET tracer candidates and their specific signal in in vivo rat autoradiography

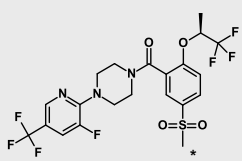

Bitopertin

0.013

3.03

4.3

0.7

97

2.1

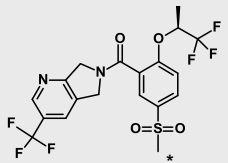

RO4993850

0.029

1.98

1.9

0.2

74

1.5

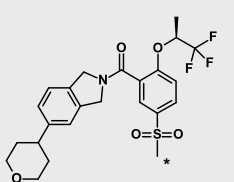

RO5013853

0.012

2.52

21

0.7

89

3.5

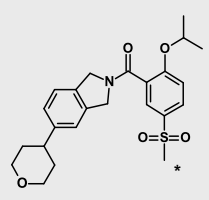

R05013852

0.017

2.23

22

0.7

88

3.2

aTB/NSB: Total Binding/ Non-Specific Binding of [3H]-ligand (measured in Medulla-Pons, rat ); ${ }^{*}$ Indicates position of tritium. 
Fig. 9. Radiosynthesis of $\left[{ }^{11} \mathrm{C}\right] \mathrm{RO} 05013852$ and $\left[{ }^{11} \mathrm{C}\right] \mathrm{RO} 05013853$ : incorporation of ${ }^{11} \mathrm{CH}_{3}$ at the last step.

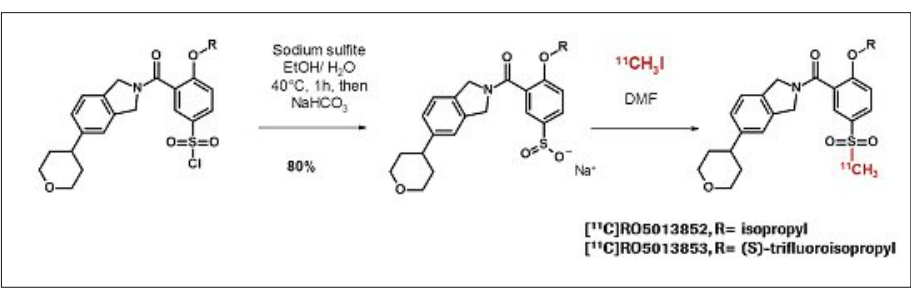

\section{Preclinical Profile of Bitopertin}

\subsection{In vitro and in vivo Pharmaco- logical Effects}

Following its discovery, bitopertin was extensively profiled in a number of in vitro and in vivo pharmacological assays. First, interestingly, detailed in vitro kinetic studies showed that bitopertin behaved as a non-competitive inhibitor with respect to the glycine binding site of the transporter and was interacting in fact, in a competitive manner with the site occupied by sarcosine derivatives such as Org-24598. [23] In rat hippocampal slice preparations, bitopertin showed effect on long-term potentiation (LTP), a cellular mechanism known to be NMDA receptor-dependent.[24] Interestingly, in this assay, we observed an inverted U-shape concentration-response curve. Indeed, at the lower tested concentrations (30 and $100 \mathrm{nM}$ ), bitopertin increased LTP suggesting, as expected, an activation of the NMDA receptor, which likely results from an increase in the extracellular level of the co-agonist glycine. However, at a higher concentration (300 nM), bitopertin had no effect on LTP. This lack of activity observed at such high concentrations could potentially result from the cellular internalization of the NMDA-R due to an excessive release of glycine. Such an inverted U-shape concentration-response curve observed with bitopertin on LTP had in fact been reported earlier with another selective GlyT1 inhibitor, CP-802,079. [24] As LTP is one of the major cellular mechanism that underlies synaptic plasticity, learning and memory, the in vitro effect observed on LTP suggested that bitopertin had potential in promoting cognitive processes. Microdialysis experiments in freely moving rats showed that bitopertin dose-dependently increased brain extracellular glycine levels with a plateau reached at about 2.3 fold over basal levels. Interestingly, an almost identical increase of glycine was measured at the same doses in the rat CSF, suggesting that CSF glycine could provide a useful clinical biomarker for measuring the pharmacodynamic effect of bitopertin in the human CNS.[23] Bitopertin was found to show behavioural effects in several naïve and pharmacologically challenged animal models. In addition to its robust effect in the L-687,414-induced hyperlocomotion assay ( $\mathrm{ID}_{50}=0.5 \mathrm{mg} / \mathrm{kg}$ po, vide supra), bitopertin prevented the hyperlocomotion induced by amphetamine in mice, a conventional dopaminergic-based model used to assess the ability of compounds to treat positive symptoms in schizophrenia. In this experiment, the maximal effect was observed at a low dose: $0.3 \mathrm{mg} / \mathrm{kg}$ and not at higher doses ( 1 and $3 \mathrm{mg} / \mathrm{kg}$ ) implying the presence of an optimal dose range to reach efficacy. Interestingly, the effect observed with bitopertin in this model suggested that dopaminergic transmission in the brain could be modulated through inhibition of GlyT1 and without direct action at dopamine D2 receptors. Bitopertin was also found to reduce the hypersensitivity to amphetamine challenge in rat sub-chroniblocker PCP, an animal paradigm mimicking the exaggerated amphetamine sensitivity typically observed in patients with schizophrenia. Thus, these results nicely suggested that bitopertin could correct a change of dopaminergic activity in the brain caused by NMDA receptor hypofunction. Furthermore, in the cognition delayed-match-to-sample (DMTS) model conducted in naïve non-human primates, bitopertin improved performance, suggesting that blockade of GlyT1 enhanced prefrontal cortex activity, a function known to be impaired in patients with schizophrenia that may contribute to the formation of cognitive and negative symptoms. Again, an inverting U-shape dose response curve was observed in this preclinical study. ${ }^{[25]}$ Finally, on the safety side, importantly, unlike the first described sarcosine-based GlyT1 inhibitors (vide supra), bitopertin did not show effects on locomotion, nor effects on respiration in rodents, which were considered of toxicological relevance. Overall bitopertin was found to be safe and well-tolerated in the preclinical toxicological studies we conducted.

\subsection{Receptor Occupancy/Efficacy Relationship in Preclinical Studies}

Having established with our PET tracer, the plasma/receptor occupancy relationship in the two preclinical species, rat and monkey (vide supra), we could determine the GlyT1 target engagement needed for bitopertin to attain efficacy in the behavioural assays described above. It is of note that the plasma concentrations of bitopercally treated with the selective NMDA-R tin measured at active doses in the rodent L-687,414/amphetamine-induced hyperlocomotion and amphetamine-induced hypersensitivity assays, led to a maximal GlyT1 occupancy of only 50\%.[26] A similar receptor occupancy of about $40 \%$ was produced by bitopertin in the non-human primates at doses that improved performance in the DMTS task (vide supra) and in agreement of the observed inverted U-shape dose response profile, higher receptor occupancies did not lead to efficacy. ${ }^{[25]}$ In summary, we found that a low to medium target engagement of less than $50 \%$ was in fact sufficient to reach optimal effects in the tested preclinical models. These very important results strongly suggested that doses leading to a low-medium GlyT1 receptor occupancy needed to be tested in the coming phase II proof of concept study in patients with schizophrenia.

\section{Clinical Results of Bitopertin in Healthy Volunteers and in Patients with Schizophrenia}

The human clinical phase I trials of bitopertin were initiated in December 2005 in healthy volunteers. First, the single and multiple ascending dose studies showed bitopertin to be safe and well-tolerated. The measured PK parameters (Cmax, AUC, half-life) across the full range of administered doses were found in very good agreement with those predicted using the PBPK approach during preclinical development.[16] In particular, a measured terminal half-life of $40 \mathrm{~h}$ (predicted $40 \mathrm{~h}$, vide supra) confirmed that bitopertin could be administered once-daily. In a subsequent CSF biomarker study, the effect of bitopertin on central levels of glycine was evaluated in healthy male volunteers treated with four sequential dose levels (3, 10, 30, $60 \mathrm{mg}$ ) once daily for 10 days. As expected based on our preclinical data (vide supra), bitopertin was found to dose dependently increase glycine levels in the CSF as compared to baseline values. ${ }^{[18]}$ In addition, a 2.3-fold increase was measured at day 10 at the highest dose tested, which, in fact was similar to the maximal increase previously observed in rats (vide supra) suggesting that, at this dose, bitopertin achieved the maximal elevation of glycine in humans. The dose-dependent increase of CSF glycine observed in this study proved the mechanism of action of bitopertin as a GlyT1 inhibitor in humans for the first time. These encouraging results were key elements in the decision to proceed with the development of bitopertin and for the choice of the clinical doses to be used in the subsequent trial in schizophrenic patients. The phase II proof-ofconcept study that started at the beginning 
of 2008 investigated the efficacy and safety profile of three dose regimens of bitopertin $(10 \mathrm{mg}, 30 \mathrm{mg}, 60 \mathrm{mg})$ in patients with schizophrenia and predominant negative symptoms who were stable on an antipsychotic treatment. The patients were treated for eight weeks with bitopertin or placebo given orally once a day. The three tested doses, 10, 30, $60 \mathrm{mg}$, were chosen to cover a broad range of CSF glycine increase, from low to high/maximal, as measured in the CSF study in healthy subjects (vide supra). Gratifyingly, at the end of 2009 , Roche reported that positive results had been obtained in this phase II proof-ofconcept study.[27] The doses of 10 and 30 mg significantly improved negative symptoms vs placebo. A trend towards functional improvement was also seen in the $10 \mathrm{mg}$ group compared to placebo. ${ }^{[28]}$ Thus at the $10 \mathrm{mg}$ dose, bitopertin demonstrated a consistent and clinically meaningful reduction in negative symptoms of schizophrenia, accompanied by the emergence of functional improvement. Efficacy parameters were improved to a lesser extent with the $30 \mathrm{mg}$ dose and no improvement in any endpoints was seen at $60 \mathrm{mg}$, the highest dose tested. ${ }^{[28]}$ Thus, fully consistent with the results obtained in preclinical models, an inverted U-shape dose efficacy relationship was also observed in patients. Furthermore, using as basis the dose/GlyT1 target engagement relationship measured in healthy volunteers (vide supra), the receptor occupancies achieved in patient by bitopertin in the phase II proof-of-concept study was estimated to be $47 \%, 67 \%$ and $77 \%$ at the 10,30 and $60 \mathrm{mg}$ dose respectively. ${ }^{[28]}$ Thus, again, fully consistent with the preclinical efficacy data, bitopertin was found to provide strongest clinical effects at a partial target occupancy of less than $50 \%$. Overall, pleasingly, a very successful translation from animal models to patients with schizophrenia appeared to operate with our GlyT1 inhibitor.[29] Noteworthy, the inverted U-shape dose response relationship as well as the partial receptor occupancy needed for efficacy appear general characteristics of this mechanism of action as these pharmacological features have since been reported for several other GlyT1 inhibitors from diverse chemical series. ${ }^{[30]}$ Bitopertin was the first potent and selective GlyT1 inhibitor to demonstrate beneficial effects on negative symptoms in patient with schizophrenia, symptoms that are, as already indicated, poorly addressed with conventional antipsychotic therapies. These results certainly generated great excitement in the scientific community, as our molecule working via a novel mechanism of action was seen as a possible disease changing treatment in patients with schizophrenia. ${ }^{[31]}$ These highly encouraging results then prompted Roche to initiate a comprehensive clinical Phase III program named Searchlyte to investigate bitopertin in combination with second-generation antipsychotics in patients with predominant negative symptoms in three double-blind, placebo-controlled, multicentre, studies: SunLyte, DayLyte, FlashLyte; 24 week treatment and, in addition, in three double-blind, placebo-controlled, multicentre studies in patients with sub-optimally controlled positive symptoms (named: TwiLyte, NightLyte, MoonLyte; 12 week treatment). In each of the six studies, two doses were tested: 10 and $20 \mathrm{mg}$, once daily for SunLyte, FlashLyte, TwiLyte, and NightLyte and 5 and $10 \mathrm{mg}$ once daily for DayLyte, and MoonLyte. Disappointingly, in the DayLyte and FlashLyte negative symptoms studies, bitopertin did not achieve significant separation from placebo. In addition, the third study (SunLyte) met the prespecified futility criteria at the planned interim analysis and was then stopped. ${ }^{[32]}$ The promising phase II proof of concept results could thus not be confirmed in the more extended phase III program. A detailed analysis revealed that both a substantially higher placebo response rate than observed in the phase II trial and an overall weak effect of bitopertin were important factor contributing to these negative results. ${ }^{[32]}$ It is noteworthy that the phase II trials of two other GlyT1 inhibitors, Org25935 [33] and $A M G 747^{[30 c]}$ reported results that were also negative with regard to primary outcome measures but were very consistent with results in our phase II trial when the same outcome measure was used. These results not only support the notion that GlyT1 inhibitor seem to have a modest effect on negative symptoms but more importantly point to the troubling issue that a strong placebo response is very common in clinical trials in patients with schizophrenia making the assessment of novel agents particularly challenging. ${ }^{[34]}$ In the sub-optimally controlled positive symptoms studies, bitopertin could achieve a significant effect over placebo in only one of the three studies (NightLyte) and at only one dose $(10 \mathrm{mg}){ }^{[35]}$ Of the two other studies, one (TwiLyte) did not differ from placebo and the other one (MoonLyte) was prematurely discontinued on the basis of results from futility analyses. Altogether, the disappointing results obtained in both the negative symptoms and the sub-optimally controlled positive symptoms studies led Roche to decide, in 2014, to stop further clinical development of bitopertin for schizophrenia. Although our clinical results challenge GlyT1 inhibition as a valid mechanism to treat negative symptoms, this mode of action continues to receive attention from others in particular for addressing cognitive symptoms. Indeed,
Yale University has recently reported an enhancement of neuroplasticity in patients with schizophrenia with GlyT1 inhibitor PF-03463275 ${ }^{[30 \mathrm{a}]}$ and is currently conducting a phase II trial for assessing its efficacy on Cognitive Impairment Associated with Schizophrenia (CIAS) (ClinicalTrials.gov: NCT01911676) and Boehringer Ingelheim is currently in phase II with BI-425809[36] for the same indication (ClinicalTrials. gov: NCT02832037).

\section{A Second Life for Bitopertin: Evaluation for the Treatment of Hematological Disorders}

GlyT1 is expressed not only in the CNS but also in peripheral tissues, mainly in erythroid cells in humans and rats where it contributes to about $40 \%$ of the transfer of glycine, a key rate-limiting component of heme biosynthesis. ${ }^{[37]}$ Therefore, inhibition of GlyT1 in these cells is expected to affect haemoglobin $(\mathrm{Hb})$ synthesis. During the course of the preclinical and clinical development of bitopertin, indeed, the levels of haemoglobin was found to be decreased, both in animals ${ }^{[38]}$ as well as in humans. ${ }^{[28,32,35]}$ This observed on-target and dose-dependent peripheral effect, led to a reduction of $\mathrm{Hb}$ levels by up to $20 \%$ and was fully reversible upon treatment cessation. Similar results have been reported recently with a structurally distinct sarcosine-based GlyT1 inhibitor, AMG 747.[30c] Detailed mechanistic pre-clinical studies sponsored by Roche, suggested that GlyT1 inhibition could provide a promising therapeutic approach for haemoglobinaopathies such as beta-thalassemia. ${ }^{[38]}$ Beta-thalassemia is a monogenic, hereditary blood disease in human characterised by a reduced or abolished synthesis of the $\alpha$-globin chain of $\mathrm{Hb}$ that leads to an excess of free $\beta$-globin chain and a build-up of toxic hemichromes which results in reduced red blood cell survival and ultimately anaemia. In $\mathrm{Hbb}^{\text {th } 3 /+}$ mice, a genetic model for beta-thalassemia, bitopertin was shown to increase red blood cell survival, to significantly raise haemoglobin levels and to ameliorate the anaemia seen in vehicle treated animals providing a preclinical validation of the GlyT1 inhibition mode of action for this haematological disorder. ${ }^{[39]}$ Based on these promising data, Roche decided recently to initiate a phase II proof-of-mechanism study to investigate the effect of bitopertin in adult patient with non-transfusion-dependent beta-thalassemia (ClinicalTrials. gov: NCT03271541). Due to the effects on the heme biosynthesis, GlyT-1 inhibitors may also have application for other haematogical disorders such as sickle cell anaemia or iron overload syndromes such as hereditary hemochromatosis. ${ }^{[40]}$ 


\section{Conclusion}

We have reported here the eventful 17-year journey of the discovery and development of bitopertin, the most advanced GlyT1 inhibitor to date: from project initiation in 2001 to its evaluation today as a potential therapeutic agent for beta-thalassemia. Our journey was abound with excitement such as the successful phase II POC results achieved in 2010 in patients with schizophrenia, but we were also faced with great disappointments with the negative phase III data in 2014. During the discovery phase, the elements that have been, in our view, essential contributors to the successful identification of bitopertin as a potent, and safe GlyT1 inhibitor include: the identification of the highly chemically tractable and drug-like benzoylpiperazine HTS hit, the routine use of MDO assays and of the fast turnaround L-687,414-based PD assay established in the project to guide our medicinal chemistry program. During the preclinical and clinical development of bitopertin, the utilisation of the PET tracer $\left[{ }^{11} \mathrm{C}\right] \mathrm{RO} 5013853$, discovered and successfully developed in our program, has been instrumental for shedding light on the inverted U-shape dose response relationship, for the understanding of the partial target engagement of bitopertin needed for efficacy both in animal models and in clinical studies and for supporting clinical dose selection. In spite of the establishment of a robust preclinical package, a thorough understanding of PK/PD and a highly successful translation from animal models to patients in the phase II POC study, negative phase III results have nevertheless been obtained. This highlights the tremendous hurdle to establish clinical meaningful improvements in behavioural outcomes in pivotal phase III settings for patients with psychiatric disorders. Bitopertin has recently initiated a second life with the exploration of its therapeutic potential for beta-thalassemia. Noteworthy, this repurposing is supported by a well characterised effect on haemoglobin synthesis initially discovered as a peripheral safety signal (i.e. decrease of haemoglobin values) and consistently observed during the preclinical and clinical development of bitopertin. Altogether, we hope that our continuous effort in the field of GlyT1 since 2001 will, in the near future, bear fruit with the demonstration of a positive effect of bitopertin in patients suffering from hemoglobinopathies. We also hope that the knowledge we have generated and shared with the community in terms of medicinal chemistry, biology, safety, PK/PD relationship and clinical development will help to foster the discovery and development of additional GlyT1 inhibitors for the benefit of the patients. ${ }^{[41]}$

\section{Acknowledgements}

I would like to thank all my colleagues who have contributed to the GlyT1 inhibitor project at Roche, particularly those from the medicinal chemistry department for their dedicated work over many years. I would also like to warmly thank all the patients who have participated and those who are currently participating in the bitopertin clinical trials.

Received: May 30, 2018

[1] P. J. Conn, C. Tamminga, D. D. Schoepp, C. Lindsley, Mol. Interven. 2008, $8,99$.

[2] J. T. Kantrowitz, D. C. Javitt, Brain Res. Bull. 2010, 83, 108

[3] D. C. Javitt, Int. Rev. Neurobiol. 2007, 78, 69.

[4] W. Danysz, C. G. Parsons, Pharmacol. Rev. 1998, 50, 597.

[5] a) R. Bergeron, T. M. Meyer, J. T. Coyle, R. W. Greene, Proc. Natl. Acad. Sci. USA 1998, 95,15730; For two early reviews on GlyT1 inhibitors, see: b) C. Sur, G. G. Kinney, Curr. Drug Targets 2007, 8, 643; c) C. Thomsen, Drug Discov. Today: Therapeutic Strategies 2006, 3 , 539.

[6] G. Tsai, H.-Y. Lane, P. Yang, M.-Y. Chong, N. Lange, Biol. Psychiatry 2004, 55, 452.

[7] A. Brown, I. Carlyle, J. Clark, W. Hamilton, S. Gibson, G. McGarry, S. McEachen, D. Rae, S Thorn, G. Walker, Bioorg. Med. Chem. Lett 2001, 11, 2007.

[8] B. N. Atkinson, S. C. Bell, M. De Vivo, L. R. Kowalski, S. M. Lechner, V. I. Ognyanov, C. S Tham, C. Tsai, J. Jia, D. Ashton, M. A. Klitenick, Mol. Pharmacol. 2001, 60, 1414.

[9] K. Kopec, D. G. Flood, M. Gasior, B. A. W. McKenna, E. Zuvich, J. Schreiber, J. M. Salvino, J. T. Durkin, M. A. Ator, M. J. Marino, Biochem. Pharmacol, 2010, 80, 1407.

[10] D. Alberati, J.-L. Moreau, R. Mory, E. Pinard, J. G. Wettstein, Pharmacol., Biochem. and Behavior 2010, 97,185 .

[11] M. D. Tricklebank, L. J. Bristow, P. H. Hutson, P. D. Leeson, M. Rowley, K. Saywell, L. Singh, F. D. Tattersall, L. Thorn, B. J. Williams, Brit. J. Pharmacol. 1994, 113, 729 .

[12] a) D. Alberati, D. Hainzl, S. Jolidon, A. Kurt, E. Pinard, A. W. Thomas, D. Zimmerli, Bioorg. Med. Chem. Lett. 2006, 16, 4321, and reference therein; b) S. Jolidon, D. Alberati, A. Dowle, H Fischer, D. Hainzl, R. Narquizian, R. Norcross, E. Pinard, Bioorg. Med. Chem. Lett. 2008, 18, 5533.

[13] E. Pinard, D. Alberati, E. Borroni, H. Fischer, D Hainzl, S. Jolidon, J. L. Moreau, R. Narquizian, M. Nettekoven, R. D. Norcross, H. Stalder, A. W. Thomas, Bioorg. Med. Chem. Lett. 2008, 18, 5134.

[14] E. Pinard, A. Alanine, D. Alberati, M. Bender, E. Borroni, P. Bourdeaux, V. Brom, S. Burner, H. Fischer, D. Hainzl, R. Halm, N. Hauser, S. Jolidon, J. Lengyel, H.-P. Marty, T. Meyer, J.-L. Moreau, R. Mory, R. Narquizian, M. Nettekoven, R. D. Norcross, B. Puellmann, P. Schmid, S. Schmitt, H. Stalder, R. Wermuth, J. G. Wettstein, D. Zimmerli, J. Med. Chem. 2010, 53, 4603

[15] K. H. Bleicher, H. J. Boehm, K. Mueller, A. Alanine, Nature Rev. Drug Discov. 2003, 5, 369.

[16] N. Parrott, D. Hainzl, D. Alberati, C. Hofmann, R. Robson, B. Boutouyrie, M. Martin-Facklam, Clin. Pharmacokinet 2013, 52, 673.

[17] E. Pinard, A. Alberati, M. Bender, E. Borroni, V. Brom, S. Burner, H. Fischer, D. Hainzl, R. Halm, N. Hauser, Bioorganic Med. Chem. letters 2010, 20, 6960 .

[18] C. Hofmann, F. Pizzagalli, C. Boetsch, D. Alberati, L. Ereshefsky, S. Jhee, A. Patat, B. Boutouyrie-Dumont, M. Martin-Facklam, Psychopharmacology 2016, 233, 2429.

[19] E. Pinard, S. Burner, P. Cueni, T. Hartung, R. D. Norcross, P. Schmid, P. Waldmeier, G. Zielinski,
H. T. Ravert, H., D. P. Holt, R. F. Dannals, J. Labelled Comp. Radiopharm. 2011, 54, 702.

[20] E. Borroni, Y. Zhou, S. Ostrowitzki, D. Alberati, A. Kumar, D. Hainzl, T. Hartung, J. Hilton, R. F. Dannals, D. F. Wong, NeuroImage, 2013, 75, 291.

[21] D. F. Wong, S. Ostrowitzki, Y. Zhou, V. Raymont, C. Hofmann, E. Borroni, A. Kumar, N. Parkar, J R. Brašić, J. Hilton, R. F. Dannals, M. MartinFacklam, NeuroImage, 2013, 75, 282

[22] M. Martin-Facklam, F. Pizzagalli, Y. Zhou, S Ostrowitzki, V. Raymont, J. R. Brasic, N. Parkar, D. Umbricht, R. F. Dannals, R. Goldwater, D. F. Wong, Neuropsychopharmacol. 2013, 38, 504.

[23] D. Alberati, J.-L. Moreau, J. Lengyel, N. Hauser, R. Mory, E. Borroni, E. Pinard, F. Knoflach, G. Schlotterbeck, D. Hainzl, J. G. Wettstein, Neuropharmacol. 2012, 62, 1152.

[24] M. Martina, Y. Gorfinkel, S. Halman, J. A. Lowe, P. Periyalwar, C. J. Schmidt, R. Bergeron, J. Physiol. 2004, 557, 489.

[25] E. Borroni, D. F. Wong, D, Alberati, T. L. Wallace, Y. Zhou, A. Kumar, E. Pinard, D. Alberati, Schiz. Bull. 2011, 37, 296.

[26] D. Alberati, E. Borroni, J. L. Moreau, D. Hainzl E. Pinard, J. G. Wettstein, Schiz. Bull. 2011, 37, 286.

[27] see www.roche.com, Investor Update, November 10,2009

[28] D. Umbricht, D. Alberati, M. Martin-Facklam, E. Borroni, E. A. Youssef, M. Ostland, T. L. Wallace, F. Knoflach, E. Dorflinger, J. G. Wettstein, A. Bausch, G. Garibaldi, L. Santarelli, JAMA Psychiatry 2014, 71, 637.

[29] D. Alberati, Schiz. Res. 2012, 136, 1, S53.

[30] a) D. C. D'Souza, R. E. Carson, N. Driesen, J. Johannesen, M. Ranganathan, J. H. Krystal, Biol. Psychiatry 2018, in press; b) D. Eddins, T. G. Hamill, V. Puri, C. E. Cannon, J. A. Vivian, S. M. Sanabria-Bohórquez, J. J. Cook, J. A. Morrow, F Thomson, J. M. Uslaner, Psychopharmacol. 2014, 231, 511; c) E. Dunayevich, R. W Buchanan, C.Y. Chen, J. Yang, J. Nilsen, J. M Dietrich, H. Sun, S. Marder, Schiz. Res. 2017, 182, 90.

[31] C. R. Hopkins, ACS Chem. Neurosci. 2011, 2, 685.

[32] D. Bugarski-Kirola, T. Blaettler, C. Arango, W. W. Fleischhacker, G. Garibaldi, A. Wang, M Dixon, R. A. Bressan, H. Nasrallah, S. Lawrie, J. Napieralski, T. Ochi-Lohmann, C. Reid, S. R. Marder Biological Psychiatry 2017, 82, 8 .

[33] J. H. Schoemaker, W. T. Jansen, J. Schipper, A. Szegedi, J. Clin. Psychopharmacol. 2014, 34190.

[34] B. J. Kinon, A. J. Potts, S. B. Watson, Curr. Opin. Psychiatry 2011, 24, 107.

[35] D. Bugarski-Kirola, N. Iwata, S. Sameljak, C. Reid, T. Blaettler, L. Millar, T. Reis Marques, G. Garibaldi, S. Kapur, Lancet Psychiatry 2016, 3, 1115.

[36] V. Moschetti, M. Desch, S. Goetz, K. H. Liesenfeld, H. Rosenbrock, K. P. Kammerer, G. Wunderlich, S. Wind, Eur. J. Drug Metab. Pharmacokinet, 2018, 43, 239.

[37] a) P.A. King, R. B. Gunn, J Gen Physiol. 1989 , 93, 321; b) A. Felipe, O. Vinas, X. Remesar, Biosci Rep. 1990, 10, 209

[38] M. Winter, J. Funk, A. Körner, D. Alberati, F. Christen, G. Schmitt, B. Altmann, A. Pospischil, T. Singer, Exp. Heematology 2016, 44, 964

[39] A. Matte, M. Winter, E. Beneduce, E. Federti, A. Siciliano, A. Macias-Garcia, A. Iolascon, T. Singer, O. Khwaja, A. Körner, J.-J. Chen, R. Hermosilla, C. Brugnara, A. Harmeier, L. De Franceschi, 22 ${ }^{\text {nd }}$ congress of the European Hematology Association, Madrid, June 22-25, 2017.

[40] D. Alberati; A. Koerner; E. Pinard, M. Winter, WO Patent Appl. No.WO 2015165842, 2015.

[41] For two excellent recent reviews on GlyT1, see: a) C. L. Cioffi, Exp. Opin. Ther. Patents 2018, 28, 197; b) C. L. Cioffi, P. R. Guzzo, Curr. Topics Med. Chem. 2016, 16, 3404 